\title{
Dealing with a local heating effect when measuring catalytic solids in a reactor with Raman spectroscopy
}

\author{
Stan J. Tinnemans, Marianne H. F. Kox, Marco W. Sletering, T. A. (Xander) \\ Nijhuis, Tom Visser and Bert M. Weckhuysen*
}

\author{
Received 15th February 2006, Accepted 4th April 2006 \\ First published as an Advance Article on the web 19th April 2006 \\ DOI: $10.1039 / \mathrm{b602311h}$
}

In continuation of our previous work on the applicability of the $G\left(R_{\infty}\right)$ correction factor for the quantification of Raman spectra of coke during propane dehydrogenation experiments (Phys. Chem. Chem. Phys., 2005, 7, 211), research has been carried out on the potential of this correction factor for the quantification of supported metal oxides during reduction experiments. For this purpose, supported chromium oxide catalysts have been studied by combined in situ Raman and UV-Vis spectroscopy during temperature programmed reduction experiments with hydrogen as reducing agent. The goal was to quantify on-line the amount of $\mathrm{Cr}^{6+}$ in a reactor based on the measured in situ Raman spectra. During these experiments, a significant temperature effect was observed, which has been investigated in more detail with a thermal imaging technique. The results revealed a temperature 'on the spot' that can exceed $100{ }^{\circ} \mathrm{C}$. It implies that Raman spectroscopy can have a considerable effect on the local reaction conditions and explains observed inconsistencies between the in situ UV-Vis and Raman data. In order to minimize this heating effect, reduction of the laser power, mathematical matching of the spectroscopic data, a different cell design and a change in reaction conditions has been evaluated. It is demonstrated that increasing the reactor temperature is the most feasible method to solve the heating problem. Next, it allows the application of in situ Raman spectroscopy in a reliable quantitative way without the need of an internal standard.

\section{Introduction}

In previous work we have demonstrated that the amount of coke, that is formed on a $\mathrm{Cr} / \mathrm{Al}_{2} \mathrm{O}_{3}$ propane dehydrogenation catalyst, can be quantified with in situ Raman spectroscopy by using a $G\left(R_{\infty}\right)$ correction factor. ${ }^{1}$ This factor can be derived from the following expressions: ${ }^{2-4}$

$$
\begin{gathered}
\Psi_{\infty}=\frac{\rho I_{0}}{s} \cdot G\left(R_{\infty}\right) \\
G\left(R_{\infty}\right)=\frac{R_{\infty}\left(1+R_{\infty}\right)}{\left(1-R_{\infty}\right)}
\end{gathered}
$$

In eqn (1) $\Psi_{\infty}$ represents the observed Raman intensity for a powdered sample of infinite thickness, $I_{0}$ the exciting Raman laser intensity, $\rho$ the coefficient of Raman generation and $s$ the scattering coefficient. The equation is valid based on the assumption that the scattering of the solid $s$ does not change. This implies e.g. that the catalyst particles may not aggregate during reaction leaving the scattering coefficient $s$ unaltered. $G\left(R_{\infty}\right)$ can then be directly determined via eqn (2) by measuring $R_{\infty}$ with diffuse reflectance spectroscopy in the UV-VisNIR spectroscopy. This $G\left(R_{\infty}\right)$ factor can be directly determined with in situ UV-Vis spectroscopy using a previously

Inorganic Chemistry and Catalysis, Department of Chemistry, Utrecht University, Sorbonnelaan 16, 3508 TB Utrecht, The Netherlands. E-mail: b.m.weckhuysen@chem.uu.nl.; Fax: +31 30 2511027; Tel: +31302534328 developed combined Raman/UV-Vis set-up. ${ }^{5,6}$ The results turned out to be in good agreement with those obtained by applying boron nitride as an internal standard. Furthermore, they are consistent with independently conducted thermogravimetrical results along the reactor bed. At that time, we envisaged that the $G\left(R_{\infty}\right)$ correction factor method should in principle work for every Raman active species present in the catalyst sample.

In order to determine the correctness of this hypothesis, we decided to investigate the temperature programmed reduction of a series of $\mathrm{Cr} / \mathrm{Al}_{2} \mathrm{O}_{3}$ catalysts in the presence of hydrogen as reducing agent. The colour of this catalyst system is known to change as a result of the reduction treatment and the conversion of $\mathrm{Cr}^{6+}$ to $\mathrm{Cr}^{3+}$ can be monitored with in situ $\mathrm{UV}-\mathrm{V}$ is spectroscopy by measuring the intensity changes of the $\mathrm{Cr}^{6+}$ charge transfer $(\mathrm{CT})$ and $\mathrm{Cr}^{3+} \mathrm{d}-\mathrm{d}$ transition bands. These spectral features should be accompanied by an intensity decrease of the characteristic $\mathrm{Cr}=\mathrm{O}\left(\mathrm{Cr}^{6+}\right)$ stretching vibration at around $996 \mathrm{~cm}^{-1}$ as measured with in situ Raman spectroscopy. The goal of this work is to show the general validity of the $G\left(R_{\infty}\right)$ correction factor for the Raman quantification of this $\mathrm{Cr}^{6+}$-species during temperature programmed reduction experiments. It will, however, be shown that a local Raman heating effect complicates this quantitative approach.

\section{Experimental}

A series of catalyst materials was prepared by the incipient wetness impregnation of alumina (Sasol, Puralox SCCA-5/ 
200, $188 \mathrm{~m}^{2} \mathrm{~g}^{-1}$ ) with $\mathrm{CrO}_{3}$ solutions with concentrations in the range of $4(0.1 \mathrm{wt} \%)$ to $61.9(1.5 \mathrm{wt} \%) \mathrm{g} \mathrm{CrO}_{3} 1^{-1}$. After impregnation the catalysts were dried for $12 \mathrm{~h}$ at $60{ }^{\circ} \mathrm{C}$ and subsequently for $12 \mathrm{~h}$ at $120{ }^{\circ} \mathrm{C}$. The catalysts were then calcined in air at $550{ }^{\circ} \mathrm{C}\left(10{ }^{\circ} \mathrm{C} \mathrm{min}{ }^{-1}\right)$ for $5 \mathrm{~h}$. After calcination, $0.1 \mathrm{wt} \%$ of boron nitride (BN, Aldrich, 99\%) was added to the catalysts as an internal standard and they were ball-milled for $20 \mathrm{~min}$ to obtain a homogeneously mixed powder. There were no indications that ball-milling had an influence on the catalyst.

For a reduction experiment typically $300 \mathrm{mg}$ of catalyst was loaded in a quartz reactor, which forms part of the combined in situ Raman/UV-Vis set-up described in previous papers. ${ }^{5,6}$ First, the catalyst was heated to $550{ }^{\circ} \mathrm{C}$ at a rate of $10{ }^{\circ} \mathrm{C}$ $\min ^{-1}$ in a mixture of $3 \mathrm{ml} \mathrm{min}{ }^{-1} \mathrm{O}_{2}$ (Hoek Loos, 99.995\%) and $12 \mathrm{ml} \mathrm{min}^{-1} \mathrm{He}$ (Hoek Loos, 99.996\%). The temperature was kept at $550{ }^{\circ} \mathrm{C}$ for $5 \mathrm{~h}$ before cooling down to $100{ }^{\circ} \mathrm{C}$. After this drying step, He was purged to remove all $\mathrm{O}_{2}$ from the system. Subsequently, the reduction was started in parallel with on-line Raman and UV-Vis analysis, by flowing $0.5 \mathrm{ml}$ $\min ^{-1}$ of a reducing agent $\left(\mathrm{H}_{2}\right.$, Hoekloos, $\left.99.0 \%\right)$ in $39.5 \mathrm{ml}$ $\min ^{-1}$ of $\mathrm{He}$, unless otherwise stated.

UV-Vis spectra were measured using a DH2000 light source (Avantes) combined with an Avaspec 2048 CCD spectrometer (Avantes) and a BI-FL400 high temperature resistant probe (Ocean Optics). Raman spectra were recorded using a Kaiser RXN spectrometer equipped with a $532 \mathrm{~nm}$ diode laser (output power could be varied up to $60 \mathrm{~mW}$ ). The laser power was measured via an Orion PD display detector (Ophir Optronics Ltd). A 5.5" objective was used for beam focusing and collection of the scattered radiation. Typically the exposure time of the CCD camera was $2 \mathrm{~s}$ and 25 spectra were accumulated to obtain a reasonable signal to noise ratio. The spectra were analyzed with Thermo Galactic Grams AI v. 7.0 software. In addition, IR-thermography experiments were carried out with a Jade J550M InSb BB Camera (CEDIP Infrared Systems) equipped with a $50 \mathrm{~mm}$ lens and a $20 \mathrm{~mm}$ extension ring. The applied camera wavelength was $3.9 \mu \mathrm{m}$ with an estimated temperature sensitivity of $20 \mathrm{mK}$.

TPR measurements were performed using a Thermo Electron TPDRO 1100 instrument. The calcined catalyst materials were loaded in a tubular quartz reactor and flushed with $\mathrm{O}_{2}$ and calcined. Subsequently, the reactor was cooled down to RT and the gas flow was adjusted to $5 \% \mathrm{H}_{2} / \mathrm{Ar}$. The temperature was then raised from RT to $700{ }^{\circ} \mathrm{C}$ at a rate of $10{ }^{\circ} \mathrm{C}$ $\min ^{-1}$, and the content of $\mathrm{H}_{2}$ in the outlet gas was monitored with a thermoconductivity detector throughout the reduction.

\section{Results and discussion}

\section{Development of a calibration line}

A first step towards the application of a spectroscopic technique in a quantitative manner is the development of a calibration line. In Raman spectroscopy, $\mathrm{Cr}^{6+}$ has a characteristic Raman shift around $996 \mathrm{~cm}^{-1}$ originating from the $\mathrm{Cr}=\mathrm{O}$ vibration. ${ }^{7-10}$ However, from our previous work where the amount of coke has been quantified with in situ Raman spectroscopy it is known that the observed Raman intensity

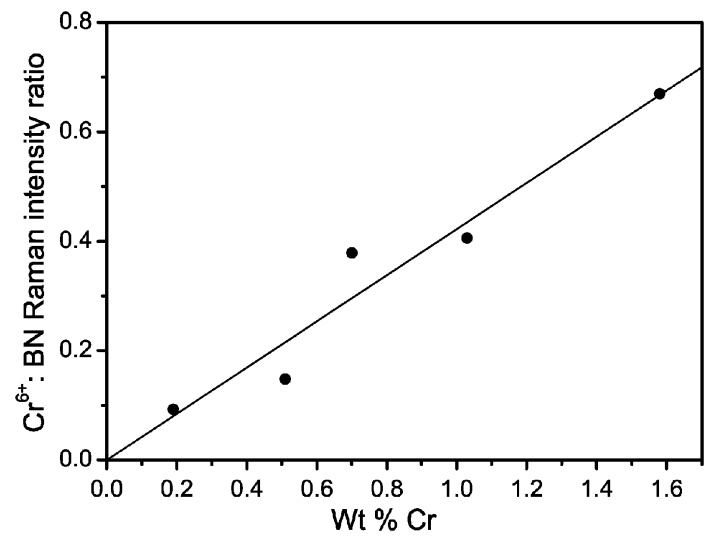

Fig. 1 Calibration line of the Raman stretching $\mathrm{Cr}=\mathrm{O}$ band plotted against the loading of a supported $\mathrm{Cr} / \mathrm{Al}_{2} \mathrm{O}_{3}$ catalyst after BN-correction $\left(y=0.42 \times, R^{2}=0.941\right)$.

is not always in agreement with the 'true' Raman intensity. ${ }^{1}$ Therefore, $\mathrm{BN}$ was added to the catalyst samples as an internal standard. The choice for BN was made because it is inert, but a strong Raman scatterer. A calibration line of the chromium band in a series of alumina-supported chromium oxide catalysts, enriched with $\mathrm{BN}$ as internal standard, differing in their chromium oxide loading is presented in Fig. 1.

Fig. 1 depicts the $\mathrm{Cr}^{6+}$ : $\mathrm{BN}$ ratio as a function of the $\mathrm{Cr}$ loading. To ensure that the catalyst remained completely oxidized, the catalyst was kept in an oxidizing environment $\left(\mathrm{O}_{2}\right.$ flow) during these measurements. Each point in the graph represents an average of six consecutive measurements. In each measurement the Raman was completely refocused. The obtained spectra were averaged, which was necessary because a change in focusing, inherent to the applied in situ set-up, resulted in slightly different $\mathrm{Cr}^{6+}$ : BN ratio's. By averaging the results of multiple spectra measured at a different focusing spot, this effect can be overcome. The straight line of Fig. 1 shows a correlation between the observed Raman intensity and the amount of $\mathrm{Cr}^{6+}$ in the catalyst in the range $0-1.5 \mathrm{wt} \%$ and suggests that the amount of $\mathrm{Cr}^{6+}$ can be monitored quantitatively by using in situ Raman spectroscopy. Recently, a similar calibration line for low-loaded supported vanadium oxide catalysts has been presented by Wu et al. ${ }^{11}$ From a practical point of view, however, it would be advantageous if no internal standard has to be added for Raman quantification. Thus, in line with the previous work, the diffuse reflectance measured at $562 \mathrm{~nm}$ with UV-Vis spectroscopy (corresponding to a Raman shift of $996 \mathrm{~cm}^{-1}$, i.e. the location of the $\mathrm{Cr}^{6+}$ band) can be used to derive the 'true' Raman intensity. ${ }^{1}$ Unfortunately, this holds only as long as the focusing of the UV-Vis-probe is constant. A difference in focusing, which is unavoidable when measuring different catalyst materials, results in a different baseline. As a consequence, the relative intensity of the $\mathrm{Cr}^{6+}$ charge transfer (CT) band around $407 \mathrm{~nm}$ changes as well. In addition, it is not possible to simply shift the baseline followed by a multiplication factor dependent on the concentration. The reason for all this is that the multiplication factor is unknown and cannot be experimentally determined. Nevertheless, within one experiment the $G\left(R_{\infty}\right)$ correction is still applicable since relative 
changes in diffuse reflectance can still be measured with $\mathrm{UV}-\mathrm{V}$ is spectroscopy.

\section{Qualitative Raman spectroscopy on supported chromium oxide catalysts during temperature programmed reduction experiments}

To prove that the use of the $G\left(R_{\infty}\right)$ correction factor is a valid approach to monitor the amount of $\mathrm{Cr}^{6+}$ during a reduction process in a catalytic reactor, a $0.5 \mathrm{wt} \% \mathrm{Cr} / \mathrm{Al}_{2} \mathrm{O}_{3}$ catalyst, enriched with $\mathrm{BN}$, was exposed to a $20 \% \mathrm{H}_{2}$ in He mixture (total flow of $15 \mathrm{ml} \mathrm{min}^{-1}$ ). The catalyst was heated in steps of $20{ }^{\circ} \mathrm{C}$ and at every temperature step, in situ Raman and $\mathrm{UV}$-Vis spectra were measured. The results of this experiment are presented in Fig. 2.

Fig. 2A shows a selection of Raman spectra measured at different temperatures during the reduction process. The reduction of $\mathrm{Cr}^{6+}$ can be monitored via the decrease of the $\mathrm{Cr}=\mathrm{O}$ band at $996 \mathrm{~cm}^{-1}$. The reduction can also be monitored via the decrease of the $\mathrm{Cr}^{6+}$ charge transfer band at around $407 \mathrm{~nm}$ and the appearance of $\mathrm{a} \mathrm{Cr}^{3+} \mathrm{d}-\mathrm{d}$ transition at around $650 \mathrm{~nm}$ (Fig. 2B). Since Fig. 2A and B provide insufficient insight in the course of the reduction profiles, this information is presented in Fig. 2C. On the left hand axis the intensity of the $\mathrm{Cr}^{6+}$ Raman band is plotted, whereas on the right hand axis the reduction with UV-Vis spectroscopy is monitored via the course of the $407 \mathrm{~nm} \mathrm{Cr}^{6+} \mathrm{CT}$ band intensity. From this figure it can be concluded that the two observed reduction temperatures differ $c a \cdot 100-150{ }^{\circ} \mathrm{C}$. For two spectroscopic techniques monitoring the same species (i.e. $\mathrm{Cr}^{6+}$ ), this is not an expected result. A plausible explanation for the observed difference in reduction temperature is the presence of a local heating effect in the laser spot. This means that UV-Vis spectroscopy must be used to verify the results obtained by Raman spectroscopy. This is clearly another advantage of combining multiple spectroscopic techniques in one set-up for studying catalytic solids at work. A similar advantage of combining techniques has recently been described for a ED-XAFS/UV-Vis set-up by Mesu et al., where beam damage was assessed when probing a catalyst system with X-rays. ${ }^{12,13}$

\section{Raman laser heating effect}

It is well-known that Raman lasers may heat a sample, especially when high laser powers are applied. ${ }^{14-20}$ To prove that a local heating effect was indeed responsible for the discrepancies between the in situ Raman and UV-Vis data, additional experiments were carried out. First of all, the same experiment was performed, but now the reduction process was stopped at $280{ }^{\circ} \mathrm{C}$. It turned out that at this temperature, the reduction process was only observed with Raman spectroscopy and not yet with UV-Vis spectroscopy. After this temperature treatment, the reactor was cooled down to room temperature and removed from the furnace under an inert gas flow (He). After removal of the reactor, a dark grey-coloured
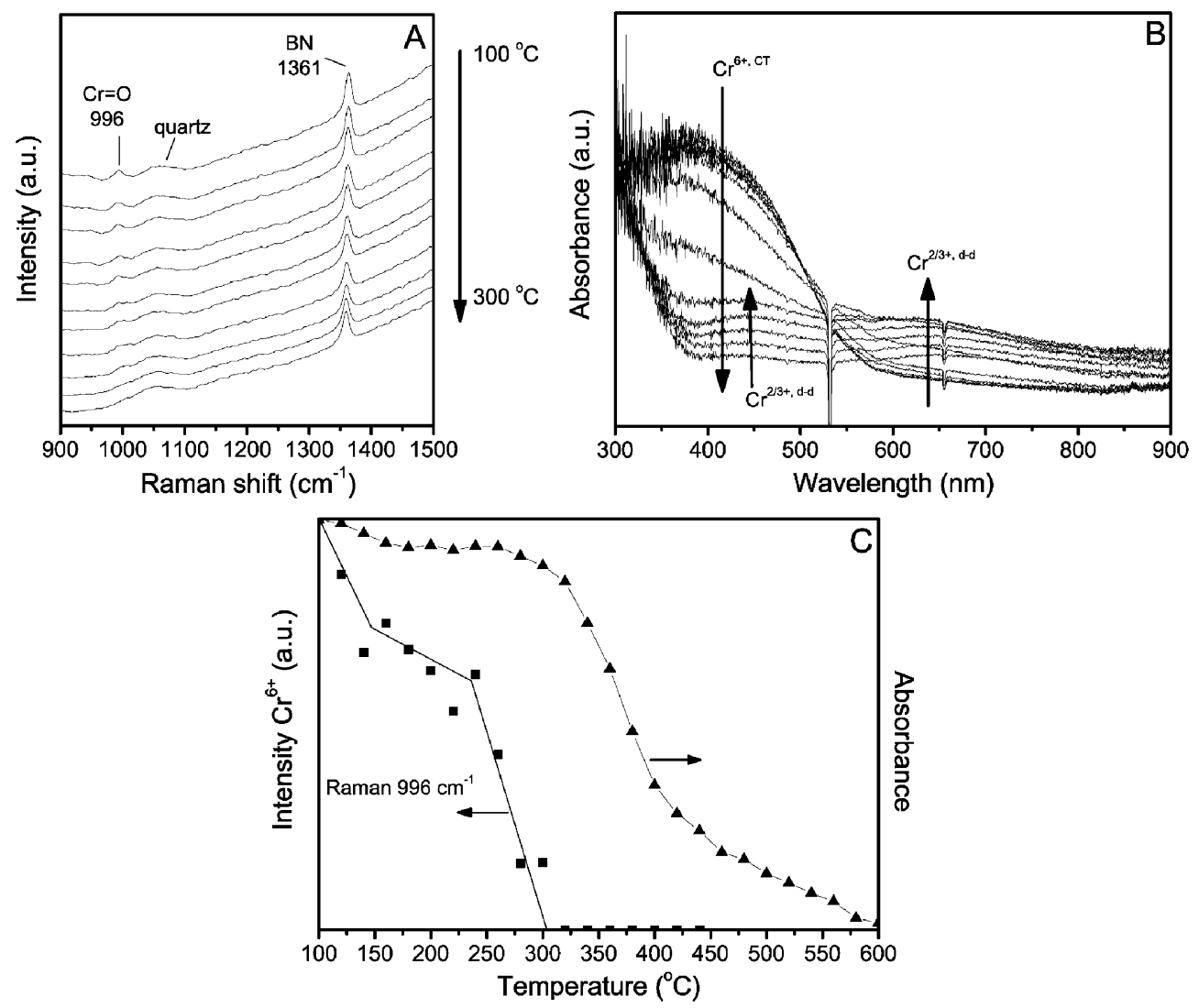

Fig. 2 Raman (A) and UV-Vis (B) spectra of a $0.5 \mathrm{wt} \% \mathrm{Cr} / \mathrm{Al}_{2} \mathrm{O}_{3}$ catalyst measured during a temperature programmed reduction experiment with $\mathrm{H}_{2}$. Fig. (C) shows the reduction profiles monitored with in situ Raman $\left(996 \mathrm{~cm}^{-1}\right.$, $\left.\boldsymbol{\square}\right)$ and UV-Vis $(407 \mathrm{~nm}, \boldsymbol{\Delta})$ spectroscopy as a function of the reduction temperature. 

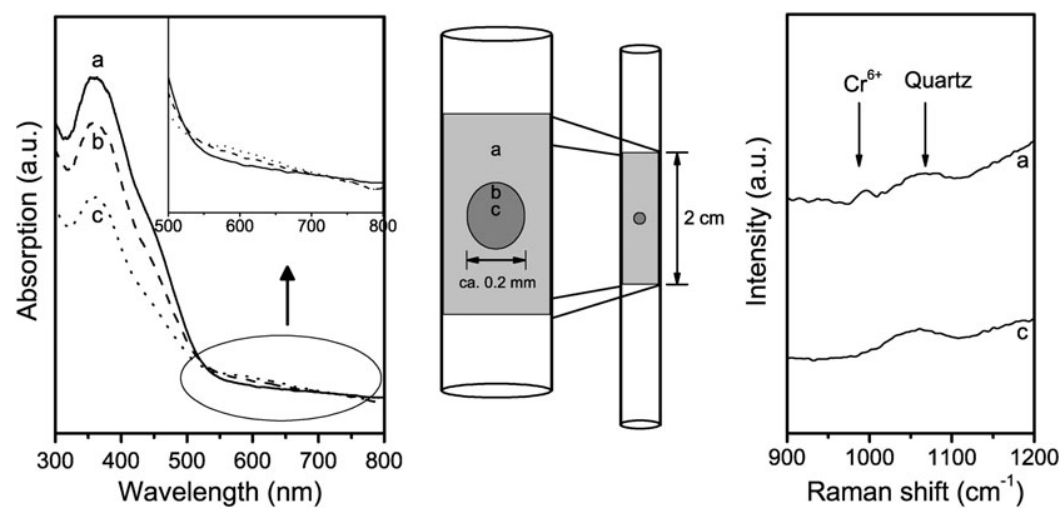

Fig. 3 UV-Vis (left) and Raman (right) spectra measured at different locations in the laser spot after reduction of a $0.5 \mathrm{wt} \% \mathrm{Cr} / \mathrm{Al}_{2} \mathrm{O}_{3}$ catalyst at $280{ }^{\circ} \mathrm{C}$.

spot was visible at the position where the Raman laser had been focused on the catalyst sample. When measuring a new Raman spectrum at a shifted position, the $\mathrm{Cr}^{6+}$ band became visible again. This is illustrated in Fig. 3.

To confirm this observation UV-Vis microspectroscopy was performed. ${ }^{21}$ In Fig. $3 \mathrm{UV}-\mathrm{Vis}$ spectra are shown, which are measured at different locations within the laser spot. Spectrum $a$ is measured in the bulk of the catalyst just outside the coloured spot, whereas spectra $b$ and $c$ are measured more towards the center of the spot. Going from spectrum $a$ to $c$ in this graph, the CT band of $\mathrm{Cr}^{6+}$ decreases in intensity, while simultaneously the $\mathrm{d}-\mathrm{d}$ transition of $\mathrm{Cr}^{3+}$ becomes visible. These UV-Vis data, in combination with the observations from Raman spectroscopy, show that at the position of the laser spot, the catalyst is reduced and that it is likely that a local temperature increase has occurred to achieve this. With this result in mind, it would be useful if the temperature inside the laserspot could be determined, and in this way correlate the UV-Vis spectra to the Raman data.

In principle, the sample temperature in a laser spot can be determined via the relative intensities of the Stokes and antiStokes shifts in a spectrum making use of Boltzmann's Law. ${ }^{22,23}$ Unfortunately, only the Stokes radiation can be measured with the available Raman spectrometer and thus the actual sample temperature in the laser spot could not be determined in this way. Instead, we have applied IR-thermography to measure the temperature of the laserspot on a completely oxidized and dehydrated catalyst sample and on a spent catalyst. For this purpose, pellets were pressed of the catalyst materials. For the (white-yellow) dehydrated catalyst, a small effect became visible $\left(c a .15{ }^{\circ} \mathrm{C}\right)$, but a much larger effect was observed obtained for the spent catalyst (having a dark grey colour). A thermographical image of this sample is presented in Fig. 4A.

The bright spot in this image clearly shows that the Raman laser is heating the catalyst sample locally, an effect which was already expected from the previous experiments. Fig. 4B was obtained when analyzing the data along the diameter of the laser spot, indicating that a local temperature increase of $70{ }^{\circ} \mathrm{C}$ is possible. Furthermore, extrapolating the slopes in these graphs, suggest that even larger temperature effects $\left(>100{ }^{\circ} \mathrm{C}\right)$ are not unlikely, in line with the data presented in Fig. $2 \mathrm{C}$.

\section{Application of quantitative Raman spectroscopy during a series of temperature programmed reduction cycles}

It will be clear from the heat effect, observed during the temperature programmed reduction experiments, that this complicates the application of the $G\left(R_{\infty}\right)$ correction factor. Recall that this factor corrects for the actual colour of the catalyst sample. In the laser spot, the catalyst will be heated and reduced and the colour consequently will change. However, simultaneously measured in situ UV-Vis spectra do not show a colour change, which is due to the lower temperature of the remaining part of the catalyst material. Therefore, it is
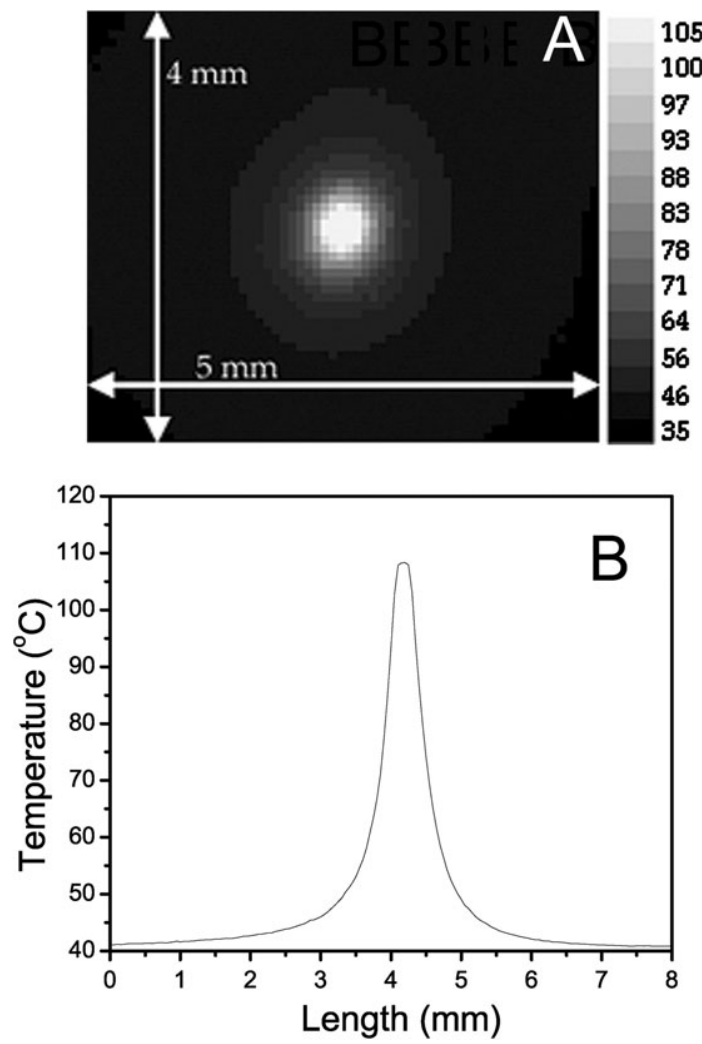

Fig. 4 (A) IR-thermographic image of a catalyst pellet after exposure to propane for $90 \mathrm{~min}$. (B) Observed temperature of the catalytic surface when a $532 \mathrm{~nm}$ laser $(50 \mathrm{~mW})$ is focused at this material. 


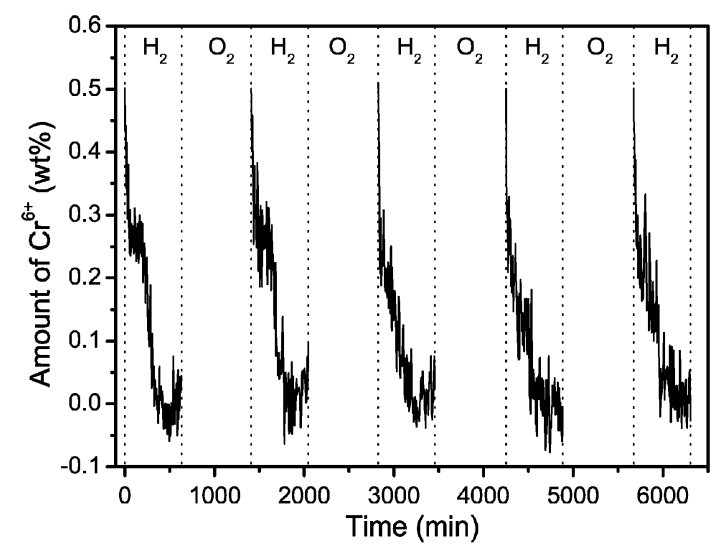

Fig. 5 The amount of $\mathrm{Cr}^{6+}$ present during five successive temperature programmed reduction cycles with $\mathrm{H}_{2}$ as reducing agent and after subsequent re-oxidation with $\mathrm{O}_{2}$ on a $0.5 \mathrm{wt} \% \mathrm{Cr} / \mathrm{Al}_{2} \mathrm{O}_{3}$ catalyst after application of the $G\left(R_{\infty}\right)$ correction factor.

not possible to calculate the $G\left(R_{\infty}\right)$ correction factor directly. This problem can be overcome by 'shifting' the course of the reduction of the $\mathrm{Cr}^{6+}$ band to lower temperatures, in order to let this reduction coincide with the observed reduction in Raman spectroscopy. In other words, shift the course of the $\mathrm{Cr}^{6+}$ as observed by UV-Vis (Fig. 2C) to lower temperatures in such a way that it will overlap with the course of the observed reduction by Raman spectroscopy. In this way the observed effect of the local heating is artificially eliminated and the $G\left(R_{\infty}\right)$ correction can be applied. The result of this operation is presented in Fig. 5 for five consecutive temperature programmed reduction cycles alternated with a re-oxidation step at $500{ }^{\circ} \mathrm{C}$ with $\mathrm{O}_{2}$. In each redox cycle the temperature is raised $20{ }^{\circ} \mathrm{C}$ every $30 \mathrm{~min}$ in the range $100-500{ }^{\circ} \mathrm{C}$.

Fig. 5 shows that a gradual reduction is observed during all reduction cycles, although in the first part of every cycle (thus at lower temperatures) the reduction of supported chromium oxide species seems to be faster. $\dagger$ This could be an indication that two different chromium oxide species are probed with Raman spectroscopy, one being more easily reducible than the other. An explanation could be the presence of both monomeric and polymeric chromium oxide species, which are likely to have a different reduction behavior. This idea is in agreement with data from Weckhuysen et al. and Airaksinen et al. where both chromate and polychromates are reported to exist for $\mathrm{Cr} / \mathrm{Al}_{2} \mathrm{O}_{3}$ catalysts. ${ }^{24-26}$

TPR experiments on the same samples, however, could not prove the presence of two distinguisable species, which is in accordance with earlier studies of Kanervo and Krause. ${ }^{27}$

The data of Fig. 5 also show that the use of the $G\left(R_{\infty}\right)$ correction factor allows the determination on-line of the amount of $\mathrm{Cr}^{6+}$ during a reduction process in a reactor. The applied method (shifting the reduction profile to lower tem-

$\dagger$ It would be advantageous if the reoxidation step could also be monitored. However, after switching the gas from $\mathrm{H}_{2}$ to $\mathrm{O}_{2}$ the Raman scattering properties of the solid were dramatically changed. As a consequence, the application of a correction factor was not possible anymore. peratures) is, however, rather subjective. Therefore, it is preferable that the Raman data are not shifted, i.e. change the conditions inside the reactor in such a manner that both in situ Raman and UV-Vis data are measuring the catalyst material under identical conditions and thus eliminate the temperature effect.

\section{Exploration of experimental conditions at which the local Raman heating effect is negligible}

There are many ways of circumventing the observed local Raman heating effect. It is possible to (1) decrease the laser power, (2) defocus the Raman laser, (3) rotate the sample, (4) use a chopper or pulsed laser, (5) probe the same volume with UV-Vis and Raman spectroscopy, and (6) increase the overall reaction temperature. A selection of these methods (1,5 and 6) was investigated in the course of this study. Some comments are made on the other methods as well.

The simplest way of decreasing the local Raman heating effect is by reducing the photon-flux on the catalyst sample. As a result the difference in the observed reduction temperature in Raman and UV-Vis spectroscopy decreases. However, the laser power cannot be reduced ceaselessly as a secondary effect is taking place, which is illustrated in Fig. 6.

On reducing the laser power, the features remain the same, but the signal-to-noise ratio becomes worse. For static experiments this is not a problem, since more Raman spectra can be averaged to maintain a good signal-to-noise ratio. However, for in in situ experiments, this will increase the time necessary for measuring one Raman spectrum and as a result dynamic information on the catalyst behavior might be lost.

Closely related to this method is a defocusing of the Raman laser. In this way the photons are spread on a larger surface, but also at the expense of the signal-to-noise ratio. This method has been applied by MacDonald and co-workers. ${ }^{28}$ Other methods to decrease the photon flux on the sample are using a chopper, a pulsed laser or moving the sample around in the focus spot of the beam. A rotating disk is an example, which can be used to prevent beam damage. ${ }^{29,30}$ However, for the developed Raman/UV-Vis set-up sample spinning is not an



Fig. 6 Raman spectra of a $0.5 \mathrm{wt} \% \mathrm{Cr} / \mathrm{Al}_{2} \mathrm{O}_{3}$ catalyst measured with an exposure time of $1 \mathrm{~s}$. 10 spectra were averaged in order to have $\mathrm{S} / \mathrm{N}$ improvement: (a) $35 \mathrm{~mW}$, (b) $20 \mathrm{~mW}$, (c) $10 \mathrm{~mW}$ and (d) $5 \mathrm{~mW}$. The spectra are normalized to the $\mathrm{BN}$ band. 

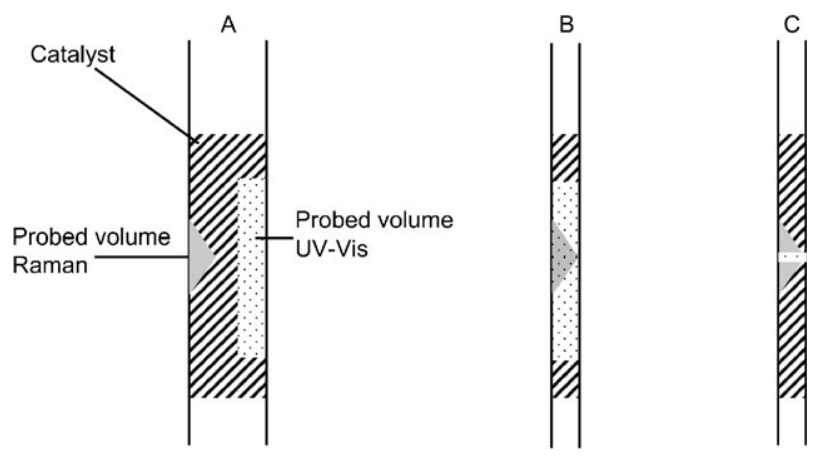

Fig. 7 Schematic representation of the probed catalyst volume with (A) a 'normal' reactor $(\varnothing=6 \mathrm{~mm})$ and a standard UV-Vis probe (400 $\mu \mathrm{m}$ fibres), (B) a reactor with a smaller inner diameter $(\varnothing=1$ $\mathrm{mm})$ and a 'standard' UV-Vis probe (400 $\mu \mathrm{m}$ fibres) and (C) a reactor with a smaller inner diameter $(\varnothing=1 \mathrm{~mm})$ and a micro-UV-Vis probe (100 $\mu \mathrm{m}$ fibres).

option and other methods for dealing with the local heat effect have to be explored.

A possibility might be changing the reactor configuration. In the normally used reactor tubes, the probed volumes by Raman and UV-Vis spectroscopy do not coincide. If the internal diameter can be reduced to an extent that both techniques probe the same volume, the heat effect can be neglected and the $G\left(R_{\infty}\right)$ correction factor can be applied directly. To understand this, one needs to realize that the Raman laser will still heat the catalyst locally, but since UVVis spectroscopy is now probing the same catalyst particles, it is no longer causing differences. This is schematically depicted in Fig. 7.

As illustrated in Fig. 7A, in a normal reactor, the volume probed by UV-Vis spectroscopy is relatively far away from the volume that is probed by the Raman spectrometer. Since the heat effect of the Raman laser is very local, a temperature effect will not be detected with UV-Vis spectroscopy at the opposite side of the reactor. If the inner diameter of the reactor is reduced, as in Fig. 7B, the situation changes. The small volume probed by Raman spectroscopy is now overlapping with the sample volume probed by UV-Vis spectroscopy. However, only a very small portion of the volume probed with UV-Vis consists of reduced chromium oxide species and therefore the reduction will still be difficult te detect. Instead, if the probed volume is more or less the same for both spectroscopic techniques, the reduction, caused by the laser might be observable with UV-Vis spectroscopy (Fig. 7C).

Unfortunately, an investigation using a reactor with an internal diameter of $1 \mathrm{~mm}$ and a micro-UV-Vis probe ${ }^{31}$ showed that the difference in observed reduction temperature is still present and close to $100{ }^{\circ} \mathrm{C}$. This observation can be explained by the limited penetration depth of the laser which means that the volumes probed by Raman and UV-Vis spectroscopy are not identical. Since solid phase Raman spectroscopy is merely a surface technique (probing depth of approximately one wavelength, $0.5 \mu$ ), this would require a reactor with an inner diameter in the order of microns, but such reactor tube would easily be plugged. This means that, although it is theoretically possible to create a reactor in which

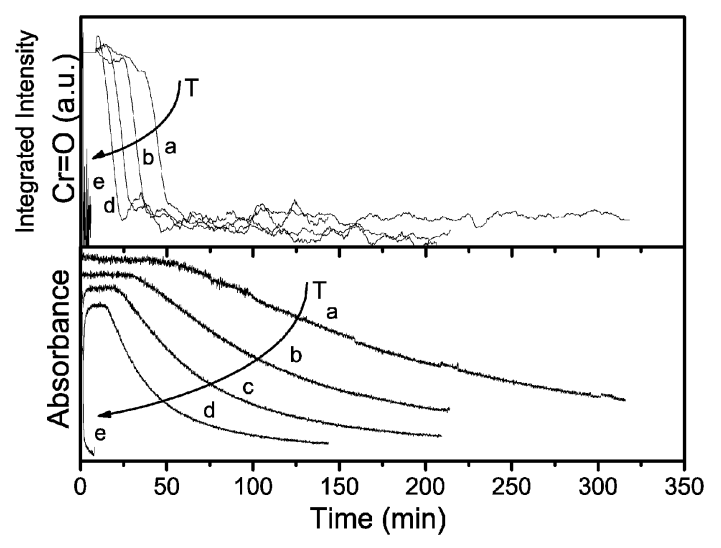

Fig. 8 Isothermal reduction profiles of a $0.5 \mathrm{wt} \% \mathrm{Cr} / \mathrm{Al}_{2} \mathrm{O}_{3}$ catalyst treated in $\mathrm{H}_{2}$ and measured at 400 (a), 420 (b), 440 (c), 460 (d) and $500{ }^{\circ} \mathrm{C}$ (e). The top figure represents the decrease of the $\mathrm{Cr}=\mathrm{O}$ band intensity observed with reduction time using in situ Raman spectroscopy, while the bottom figure illustrates the intensity profile of the $\mathrm{UV}-\mathrm{V}$ is band located at $407 \mathrm{~nm}$ as a function of the reduction time.

the temperature effect is minimized, this reactor tube could never be used for in situ experiments in a practical manner.

Finally, we studied circumventing the local heating effect by changing the reaction conditions. Since it is shown that it is necessary to get a homogeneous temperature distribution throughout the reactor tube, this means that the overall reaction temperature must be increased. The influence of the temperature of the reactor on the difference in observed reduction behavior was investigated by performing isothermal reductions with hydrogen at different temperatures in the range $400-500{ }^{\circ} \mathrm{C}$. The results of these experiments are given in Fig. 8.

Fig. 8A illustrates the course of the $\mathrm{Cr}=\mathrm{O}$ band intensity as determined with in situ Raman spectroscopy, while in Fig. 9B the course of the $407 \mathrm{~nm} \mathrm{UV-Vis} \mathrm{band} \mathrm{intensity} \mathrm{is} \mathrm{plotted.}$ Combining these two figures indicates that the difference in observed reduction temperature by UV-Vis and Raman spectroscopy decreases if the temperature of the furnace is

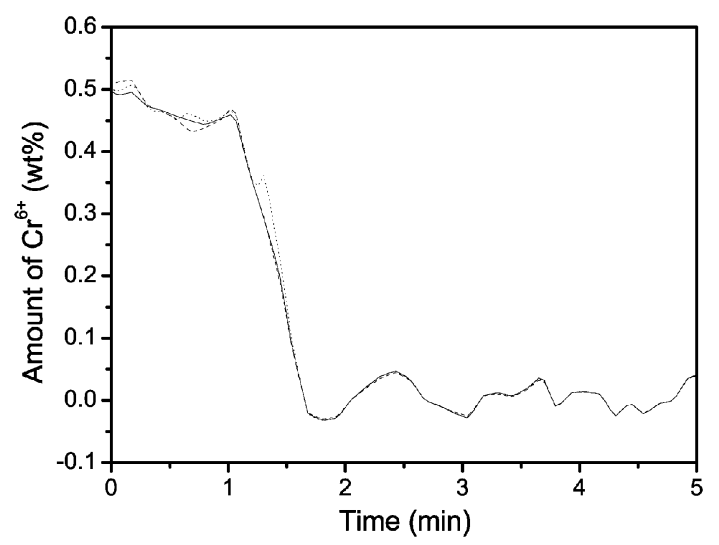

Fig. 9 Amount of $\mathrm{Cr}^{6+}$ present in a $0.5 \mathrm{wt} \% \mathrm{Cr} / \mathrm{Al}_{2} \mathrm{O}_{3}$ catalyst during a reduction with $\mathrm{H}_{2}$ at $500{ }^{\circ} \mathrm{C}$ after application of the $G\left(R_{\infty}\right)$ correction factor (dots) and the application of $\mathrm{BN}$ as internal standard (dash). The solid line represents the uncorrected data. 
increased. The results show that at relatively low temperatures the Raman heating effect is still substantial, but at temperatures close to $500{ }^{\circ} \mathrm{C}$ these differences become negligible. This also means that the application of the $G\left(R_{\infty}\right)$ correction factor is limited to high temperature reactions for this type of catalytic materials. However, differences may occur when other catalytic systems are studied with this set-up.

Finally, we have determined the amount of $\mathrm{Cr}^{6+}$ during a hydrogen reduction at $500{ }^{\circ} \mathrm{C}$ by using the $G\left(R_{\infty}\right)$ correction factor. The results are compared with those obtained when using $\mathrm{BN}$ as internal standard in Fig. 9. It is clear that both correction methods show good agreement with each other. However, a remark must be made that during the reduction of $\mathrm{Cr}^{6+}$ the relative changes occuring at $562 \mathrm{~nm}$ are rather small, and as a consequence, the influence of the $G\left(R_{\infty}\right)$ correction factor on the raw Raman data is relatively small. Nevertheless, it is shown that it is indeed possible to develop the technique of quantitative in situ Raman spectroscopy without the use of an internal standard, such as BN.

Recently, we have discussed the quantification of the amount of coke formed during a catalytic reaction as measured with in situ Raman spectroscopy. ${ }^{1}$ The methodology was based on the use of the $G\left(R_{\infty}\right)$ correction factor. It is of course important to check whether the obtained knowledge about the local Raman heating effect affects the conclusions made. As shown in this paper, the local Raman heating effect is negligible at temperatures above $500{ }^{\circ} \mathrm{C}$. Since the dehydrogenation reaction of propane is typically performed at $550{ }^{\circ} \mathrm{C}$ it can be anticipated that the Raman laser did not have any significant effect. In other words, the application of the $G\left(R_{\infty}\right)$ factor, online measured with in situ UV-Vis spectroscopy, provides an elegant way of quantifying in situ Raman spectra in a catalytic reactor as long as one can deal with the observed Raman heating effects in a satisfactory manner.

\section{Conclusions}

It is shown that a combined in situ Raman/UV-Vis set-up allows the on-line monitoring of the redox process of a supported chromium oxide catalyst during a series of temperature programmed reduction cycles. The UV-Vis spectra can be used to quantify the Raman spectra by performing a $G\left(R_{\infty}\right)$ correction with an uncertainty of less than $10 \%$. It is shown that this method is always applicable as long as the temperature is homogeneously distributed throughout the reactor bed. This is of great importance since a Raman laser is able to cause a local heating effect, which in one of our experiments resulted in a difference in observed reduction temperature of more than $100{ }^{\circ} \mathrm{C}$ for both spectroscopic techniques. In other words, a combined set-up allows with one technique (UV-Vis) to verify the results obtained with another technique (Raman).

Furthermore, it is shown that circumventing this local Raman heating effect is far from trivial as is often assumed. Reducing the laser power results in a reduced sample heating, but at the expense of the signal-to-noise ratio. If the observed reaction processes are slow, this is not really a problem. However, with fast reaction processes taking place in a reactor one might lose important information. Changing the cell design is only useful if the same sample volume can be probed by both spectroscopic techniques. In heterogeneous catalysis, this method is generally inapplicable since the required inner diameter of the reactor would cause severe reactor plugging. Increasing the overall reaction temperature clearly reduces the Raman heating effect, but limits the applicability of the experimental set-up to high-temperature catalytic reactions. It is shown that for the system under investigation the heat effect becomes negligible when the reactor temperature is close to $500{ }^{\circ} \mathrm{C}$. Nevertheless, carefully selecting the reaction parameters makes it possible to use in situ Raman spectroscopy in a quantitative manner making use of the $G\left(R_{\infty}\right)$ correction factor.

\section{Acknowledgements}

Dr P. Brémond (CEDIP Infrared Systems) and P. van Riel (L-A-P Specialty Products \& Services) are kindly acknowledged for their contribution to the IR-thermography experiments. B.M.W. acknowledges financial support from NRSCC, NWO-CW VanderLeeuw and NWO-CW/VICI.

\section{References}

1 S. J. Tinnemans, M. H. F. Kox, T. A. Nijhuis, T. Visser and B. M. Weckhuysen, Phys. Chem. Chem. Phys., 2005, 7, 211.

2 S. Kuba and H. Knozinger, J. Raman Spectrosc., 2002, 33, 325.

3 B. Schrader and G. Bergmann, Zh. Anal. Khim., 1967, 225, 230.

4 D. N. Waters, Spectrochim. Acta, Part A, 1994, 50, 1833.

5 T. A. Nijhuis, S. J. Tinnemans, T. Visser and B. M. Weckhuysen, Phys. Chem. Chem. Phys., 2003, 5, 4361.

6 T. A. Nijhuis, S. J. Tinnemans, T. Visser and B. M. Weckhuysen, Chem. Eng. Sci., 2004, 59, 5487.

7 M. A. Vuurman, F. D. Hardcastle and I. E. Wachs, J. Mol. Catal., 1993, 84, 193.

8 M. A. Vuurman, D. J. Stufkens, A. Oskam, J. A. Moulijn and F. Kapteijn, J. Mol. Catal., 1990, 60, 83.

9 B. M. Weckhuysen, I. E. Wachs and R. A. Schoonheydt, Chem. Rev., 1996, 96, 3327.

10 B. M. Weckhuysen, R. A. Schoonheydt, J. M. Jehng, I. E. Wachs, S. J. Cho, R. Ryoo, S. Kijlstra and E. Poels, J. Chem. Soc., Faraday Trans., 1995, 91, 3245.

11 Z. Wu, C. Zhang and P. C. Stair, Catal. Today, 2006, 113, 40.

12 J. G. Mesu, A. M. J. van der Eerden, F. M. F. de Groot and B. M. Weckhuysen, J. Phys. Chem. B, 2005, 109, 4042.

13 S. J. Tinnemans, J. G. Mesu, K. Kervinen, T. Visser, T. A. Nijhuis, A. M. Beale, D. E. Keller, A. M. J. van der Eerden and B. M. Weckhuysen, Catal. Today, 2006, 113, 3.

14 B. T. Bowie, D. B. Chase and P. R. Griffiths, Appl. Spectrosc., $2000,54,164 \mathrm{~A}$

15 N. J. Everall, J. Lumsdon and D. J. Christopher, Carbon, 1991, 29, 133.

16 J. Johansson, S. Pettersson and L. S. Taylor, J. Pharm. Biomed. Anal., 2002, 30, 1223.

17 H. Kagi, I. Tsuchida, M. Wakatsuki, K. Takahashi, N. Kamimura, K. Iuchi and H. Wada, Geochim. Cosmochim. Acta, 1994, 58, 3527.

18 A. Paolone, A. Sacchetti, T. Corridoni, P. Postorino, R. Cantelli, G. Rousse and C. Masquelier, Solid State Ionics, 2004, 170, 135.

19 B. Schrader, A. Hoffmann and S. Keller, Spectrochim. Acta, Part A, 1991, 47, 1135.

20 O. N. Shebanova and P. Lazor, J. Raman Spectrosc., 2003, 34, 845.

21 L. G. van de Water, J. A. Bergwerff, T. A. Nijhuis, K. P. de Jong and B. M. Weckhuysen, J. Am. Chem. Soc., 2005, 127, 5024.

22 D. A. Long, Raman Spectroscopy, McGraw-Hill Inc., UK, 1977.

23 B. J. Kip and R. J. Meier, Appl. Spectrosc., 1990, 44, 707.

24 B. M. Weckhuysen, A. A. Verberckmoes, A. L. Buttiens and R. A. Schoonheydt, J. Phys. Chem., 1994, 98, 579. 
25 B. M. Weckhuysen, L. M. De Ridder and R. A. Schoonheydt, $J$. Phys. Chem., 1993, 97, 4756.

26 S. M. K. Airaksinen, A. O. I. Krause, J. Sainio, J. Lahtinen, K. J. Chao, M. O. Guerrero-Perez and M. A. Banares, Phys. Chem. Chem. Phys., 2003, 5, 4371.

27 J. M. Kanervo and A. O. I. Krause, J. Catal., 2002, 207, 57.
28 A. M. Macdonald, A. S. Vaughan and P. Wyeth, J. Raman Spectrosc., 2005, 36, 185.

29 W. Kiefer and H. J. Bernstein, Appl. Spectrosc., 1971, 25, 500.

30 H. J. Sloane and R. B. Cook, Appl. Spectrosc., 1972, 26, 589.

31 A. M. Beale, A. M. J. Van der Eerden, K. Kervinen, M. A. Newton and B. M. Weckhuysen, Chem. Commun., 2005, 24, 3015. 